\title{
Research on Robust Adaptive and Efficient Control System: A Theoretical Approach
}

\author{
Fengling Yin and Bingquan Huo \\ Binzhou Polytechnic, Shandong, China \\ yinfengling111@126.com
}

\begin{abstract}
In this paper, a robust adaptive repetitive control algorithm is presented for periodically time-varying systems. Periodic time-varying parameter estimation through periodic learning algorithm, and the uncertainty of aperiodic was robust adaptive method. Different from the existing repetitive control, this paper introduces the design of a new variable cycle number control. Convergence error when the number increase will gradually decrease due to the cyclical repetition character system, in order to ensure the global asymptotic stability. Further, this method is applied to a class of nonlinearly parameterized systems with non-parametric disturbances, and the tracking error converges asymptotically. The results verify the simulation model of the inverse pendulum. In addition, it is proved that the proposed design method is applied to eliminate the influence of approximation error of neural network. Theoretical analysis shows that the system output is convergent to the desired one and all signals in the network based robust adaptive repetitive control system are bounded. The experimental result illustrates the effectiveness of our proposed methodology.
\end{abstract}

Index terms: Neural Network, Control System, Robust Approach, Non-linear Analysis

\section{Introduction}

Periodic motion phenomenon is widespread in the control system, such as hard drive, cd-rom drive system, pulse width modulation (PWM) inverter in the power electronics industry, and uninterrupted power supply system, linear motor system and so on. The dynamic characteristics of these systems often contain periodic time-varying coefficient, this paper will such a system is called periodic time-varying system. There are a variety of analysis methods to deal with periodic time-varying systems. In [1], Hill equation is used to describe differential equations with periodic coefficient, and analyze its stability, Floquet-yapunov transformation in literature $[2,3]$, the continuous time and discrete time system is the stability of the system theory, respectively is converted into a constant parameter of the form of differential equation and difference equation, the literature $[4,5)$ of the periodic function itself by Fourier series approximation, an adaptive law is by differential Fourier coefficient to estimate, the constant parameterization method is also used in the back-stepping design cycle system [6] for some systems with periodic interference, literature [7] the adaptive feedforward offset technology to eliminate interference signals in sine function expression, this method is proved with repetitive control are equivalent, literature [8] half cycle of integral operator and delay operator is applied to deal with the half cycle symmetric disturbance signal estimation problem, literature [9] settled through nonlinear controller is designed for a class of linear systems complete inhibition of triangular wave interference. With the Floquet theory and stationary parameterized indirect methods, such as different, this article adopts the repetitive control of a more direct method, using cycle correction law of periodic signal are estimated directly. 
Repetitive control [10] is to realize the interference suppression periodic signal tracking, cycle and the cycle parameters of the approaching precision control technology. The classic theory of repetitive control requirements within the system contains the same implementation mechanism of periodic signal, which is based on the analysis and design of the internal model principle. With the development of modern control theory, the method based on Lyapunov like the 16th [11] repeat the analysis of the control system theory, the design of the repetitive controller is more intuitive from the frequency domain to the time domain, not only provide the convenience for designers, made it possible for combining with other advanced control technology. The literature [17] combined repetitive control and sliding mode control, under the condition of the system of upper and lower bounds of the uncertainty has, repeat variable structure controller, get the asymptotic stability of the system; Further, when the upper unknown, introducing the forgetting factor, adaptive repetitive controller is designed, and prove the uniform boundedness of the variables of the system. The literature [18] a part of the saturated learning algorithm, the global stability of the system, and this method was applied to robotic system, realized the system output to the desired trajectory tracking. The literature [19] and literature [20] are based on the system of the parametric uncertainties, and the difference is: the former belongs to the linear parameterization, while the latter is also carried out nonlinear parametric. Parameterized periodic time-varying parameters through learning cycle after the law is discussed.

To sum up, the repetitive control can effectively deal with periodic time-varying coefficient, for the cycle system contains aperiodic uncertainty, also should be combined with other control methods, such as adaptive control process can be parameterized uncertainties. The robust adaptive control method is another kind of form is to join in the control law for the compensation to the disturbance of items, in the system interference unknown circumstances, increase the adaptive estimation of law, guarantee the system stability [29], but as a result of the controller to join the symbol function, flutter phenomenon in a system. The literature [23] using hyperbolic function replacement symbol function, to avoid the chatter, but can only get boundedness of system variables. This article in view of the periodic time-varying system, a robust adaptive repetitive control method is put forward, the method using the learning law directly estimation periodic time-varying parameters, and combining the method of robust adaptive processing aperiodic uncertainty, in the controller design, using the hyperbolic function replacement symbol function, in order to avoid chatter. Unlike literature [23], introduced in the hyperbolic function series convergent sequence, the sequence of the denominator for the number of cycle power and repetitive control existing in the controller design using the cycle count, make circles of approximation error with the increase of cycle number and gradually reduce, global asymptotic stability of the system from theory. Further, the method was applied to a class of nonlinear parametric system which makes the system. In non-parametric perturbation circumstances, still output error converges to zero. Finally, the method is used to eliminate the approximation errors of the neural network to the influence of the repetitive control system, proved that the system all variables of boundedness and asymptotic convergence of the output error. Simulation examples use inverted pendulum model and mechanical arm model and the method is proven to be effective to deal with periodic time-varying system, the system has good tracking performance.

\section{General Analysis of the Problem}

First of all, we give an adaptive estimation method, considering the conventional firstorder nonlinear system expressed as the formula 1 and 2:

$$
\dot{x}=f(x)+g(x) u+\theta^{*} \xi(x)+\eta(x, t)
$$




$$
\dot{x}_{N}=f_{N}\left(x_{N}, u, \theta\right) \equiv f\left(x_{N}\right)+g\left(x_{N}\right) u+\theta^{*} \xi\left(x_{N}\right)
$$

System (1) at the same time with parameter uncertainty and non-parametric uncertainty, these uncertainties can be caused by many factors, such as the measurement noise and model error and external disturbance, etc. Assumptions:

$$
|\eta(x, t)| \leq \alpha^{*} \rho(x), \forall x \in R, \forall t \in R^{+}
$$

Robust stability for such global problems, the design of feedback control law and the system is global asymptotic stability at the origin, such as control law:

$$
u=-\frac{1}{g(x)}\left[k_{1} x+f(x)+\theta \xi(x)+\eta(x, \alpha)\right]
$$

The traditional adaptive control method design of [30] is given as follows:

$$
\begin{gathered}
\eta(x, \alpha)=\alpha \rho(x) \operatorname{sgn}(x) \\
\dot{\theta}(t)=\gamma_{1} x \xi(x), \quad \gamma_{1}>0 \\
\dot{\alpha}(t)=\gamma_{2}|x| \rho(x), \quad \gamma_{2}>0
\end{gathered}
$$

The defect of this kind of controller are: 1) type (5) the symbol function can bring flutter to system; 2) due to the actual operation, the system steady-state error can't be accurate to $0,(7)$ the right hand side of type $\alpha$ constant greater than zero, then A will continue to accumulate and increasing; 3$) \eta(x, t)$ function is discontinuous, unable to derivation, the back-stepping design does not apply to strict feedback nonlinear systems. In view of this, the literature [23] gives a smooth boundary estimation method, the design is as follows:

$$
\begin{gathered}
\eta(x, \alpha)=\alpha w(x) \\
\dot{\theta}(t)=\gamma_{1}\left[x \xi(x)-\sigma_{1}\left(\theta-\theta^{0}\right)\right] \\
\dot{\alpha}(t)=\gamma_{2}\left[x w(x)-\sigma_{2}\left(\alpha-\alpha^{0}\right)\right] \\
w(x)=\rho(x) \tanh \left(\frac{x \rho(x)}{\varepsilon}\right)
\end{gathered}
$$

Of course, we also can take the world deal with the parameter estimation methods, but if we suppose is $\theta(t)$ cycle, a new system model is as follows:

$$
\dot{x}=f(x)+g(x) u+\theta(t) \xi(x)+\eta(x, t)
$$

In the above formula, with $\theta(t)$ bound estimation method to deal with time-varying parameter is too conservative. In view of the repetitive control can effectively deal with periodic time-varying parameters, we adopt cycle study law estimate $\theta(t)$, and introducing new variables-in the processing of cycle number named $j=[t / T]$. For the controller design and convergence analysis of needs, gives two definitions and a lemma.

Definition 1: Series convergent sequence:

$$
\varepsilon^{j}=\frac{q}{j^{l}}, \quad j=1,2,3, \ldots, q
$$

The $\varepsilon^{j}$ should hold the following properties: 


$$
\begin{array}{r}
\lim _{j \rightarrow \infty} \varepsilon^{j}=0 \\
\lim _{j \rightarrow \infty} \sum_{i=0}^{j} \varepsilon^{j}<\infty
\end{array}
$$

Definition 2: As for scalar $(a)$, limiting function $s a t_{\bar{\alpha}}(a)$ is defined as:

$$
\operatorname{sat}_{\bar{\alpha}}(a)=\left\{\begin{array}{lc}
\bar{\alpha}^{1} & a<\bar{\alpha}^{1} \\
a & \bar{\alpha}^{1} \leq a \leq \bar{\alpha}^{2} \\
\bar{\alpha}^{2} & a>\bar{\alpha}^{2}
\end{array}\right.
$$

Lemma one is expressed in formula 17 and 18:

$$
\begin{gathered}
\left(a_{1}-\operatorname{sat}_{\bar{a}}\left(a_{2}\right)\right)^{2} \leq\left(a_{1}-a_{2}\right)^{2} \\
\left(a_{1}-\operatorname{sat}_{\bar{a}}\left(a_{2}\right)\right)\left(a_{2}-\operatorname{sat}_{\bar{a}}\left(a_{2}\right)\right) \leq 0
\end{gathered}
$$

In [23] controller design while avoiding the symbolic function of defects, but also at the expense of the asymptotic stability of the system; [31] the arctangent function used in the design of the controller, but also can achieve the bounded convergence. The control goal is to find the appropriate controller and the system (12) is the global asymptotic stability.

\section{Our Theoretical Analysis}

In view of the system (12), the design of feedback controller is formulated as:

$$
u=-\frac{1}{g(x)}\left(k_{2} x+f(x)+\theta \xi(x)+\alpha \rho(x) \tanh \left(\frac{x \rho(x)}{\varepsilon^{j}}\right)\right)
$$

The adaptive estimator circle is denoted as:

$$
\dot{\hat{\alpha}}=-\gamma_{3} \varepsilon^{j} \alpha+\gamma_{3} x \rho(x) \tanh \left(\frac{x \rho(x)}{\varepsilon^{j}}\right)
$$

1) Part of limiting learning law:

$$
\theta(t)=\left\{\begin{array}{cc}
\operatorname{sat}_{\bar{\theta}}(\theta(t-T))+\gamma_{4} x \xi(x), & t \in[0, \infty) \\
0, & t \in[-T, 0)
\end{array}\right.
$$

2) All the limiting law of learning:

$$
\begin{aligned}
& \theta^{*}(t)=\left\{\begin{array}{cc}
(\theta(t-T))+\gamma_{4} x \xi(x), & t \in[0, \infty) \\
0, & t \in[-T, 0)
\end{array}\right. \\
& \theta(t)=\operatorname{sat}_{\bar{\theta}}\left(\theta^{*}(t)\right)
\end{aligned}
$$

Notation: the usage of $\varepsilon^{j}$ is to: 1) make the error bounds with the increase of cycle number and decline; 2) type (20) on the right-hand side first make circles estimate is not too big. Therefore, this method guarantees global asymptotic stability of the system. But due to the nature of type (14), when the cycle number tends to infinity, $\tanh \left(\frac{x \rho(x)}{\varepsilon^{j}}\right)$ 
tends to sign function, so the emergence of chatter at infinity; but in the actual operation, time will not tends to infinity, as long as the proper adjustment of the parameters Q and 1, can avoid the flutter. Literature [20] has proved using partial limiting learning law and all limiting learning law can make the system stable, where the periodic law of learning (21) and (22) can guarantee that the parameter estimation is bounded, and guarantee the stability of the system.

Theorem 1: The system (12) in $\eta(x, t)$ type (3), meet in the feedback control law (19) and the parameter adaptive law (20) under the action of respectively using part of limiting law of learning (21) or limiting law of learning (22), to ensure the system is global asymptotic stability can.

Proof: The controller (19) into the system (1), we derive:

$$
\begin{aligned}
& \dot{x}=-k_{2} x+\tilde{\theta}(t) \xi(x)-\hat{\alpha} \rho(x) \tanh \left(\frac{x \rho(x)}{\varepsilon^{j}}\right)+\eta(x, t) \\
& V(t)=V_{1}+V_{2}+V_{3} \\
& V_{1}=\frac{1}{2} x^{2} \\
& V_{2}=\frac{1}{2 \gamma_{3}} \alpha^{2} \\
& V_{3}=\frac{1}{2 \gamma_{4}} \int_{t-T}^{t} \theta^{2} d \tau \\
& \dot{V}_{1}=x\left(-k_{2} x+\theta(t) \xi(x)-\alpha \rho(x) \tanh \left(\frac{x \rho(x)}{\varepsilon^{j}}\right)+\eta(x, t)\right)= \\
& -k_{2} x^{2}+\theta(t) \xi(x) x+x \eta(x, t)-\alpha \rho(x) \tanh \left(\frac{x \rho(x)}{\varepsilon^{j}}\right) \\
& \dot{V}_{2}=\frac{1}{\gamma_{3}} \alpha\left(\gamma_{3} \varepsilon^{j} \alpha-\gamma_{3} x \rho(x) \tanh \left(\frac{x \rho(x)}{\varepsilon^{j}}\right)\right)= \\
& \alpha \varepsilon^{j} \alpha-\alpha x \rho(x) \tanh \left(\frac{x \rho(x)}{\varepsilon^{j}}\right) \\
& \Delta V_{3}=\frac{1}{2 \gamma_{4}} \int_{t-T}^{t}\left(\theta^{2}(\tau)-\theta^{2}(\tau-T)\right) d \tau \leq \int_{t-T}^{t}-x \xi(x) \theta(\tau) d \tau \\
& \Delta V_{3}=\frac{1}{2 \gamma_{4}} \int_{t-T}^{t}\left(\theta^{2}(\tau)-\theta^{2}(\tau-T)\right) d \tau \leq \frac{1}{\gamma_{4}} \int_{t-T}^{t}(\theta(\tau-T)-\theta(\tau)) d \tau \\
& \leq \frac{1}{\gamma_{4}} \int_{t-T}^{t}\left(\theta^{*}-\operatorname{sat}_{\bar{\theta}}\left(\theta^{*}\right)\right)\left(\theta-\operatorname{sat}_{\bar{\theta}}\left(\theta^{*}\right)\right) d \tau-\int_{t-T}^{t} x \xi(x) \theta d \tau \\
& \Delta V_{3}=V_{3}(t)-V_{3}(t-T) \leq \int_{t-T}^{t}-x \xi(x) \theta(\tau) d \tau
\end{aligned}
$$




$$
\begin{gathered}
\Delta V(t)=V(t)-V(t-T)=\int_{t-T}^{t}\left(\dot{V}_{1}+\dot{V}_{2}\right) d \tau+\Delta V_{3}(t) \leq \\
\int_{t-T}^{t}\left(-k_{2} x^{2}-\alpha^{*} x \rho(x) \tanh \left(\frac{x \rho(x)}{\varepsilon^{j}}\right)+\alpha \varepsilon^{j} \alpha+\alpha^{*}|x| \rho(x)\right) d \tau \\
\leq \int_{t-T}^{t}\left(-k_{2} x^{2}+\left(\alpha^{*} \delta\right)+\frac{1}{4} \alpha^{* 2} \varepsilon^{j}\right) d \tau \\
V(t)=V\left(t_{0}\right)+\sum_{j=0}^{i-1} \Delta V(t-j T) \\
V(t)=V\left(t_{0}\right)+\sum_{j=0}^{i-1} \Delta V(t-j T)=V\left(t_{0}\right)- \\
k_{2} \sum_{j=0}^{i-1} \int_{t-(\mathrm{j}+1) \mathrm{T}}^{t-j T} x^{2} d \tau+\left(\alpha \times \delta+\frac{1}{4} \alpha^{* 2}\right) T \sum_{i=0}^{i-1} \varepsilon^{i-j} \\
\dot{V}(t) \leq-k_{2} x^{2}+\left(\alpha^{*} \delta+\frac{1}{4} \alpha^{* 2}\right) \varepsilon \\
V\left(t_{0}\right) \leq V(0)-k_{2} \int_{0}^{t_{0}} x^{2} d \tau \\
\lim _{t \rightarrow \infty} V(t) \leq \max _{t_{0} \in[0, T)} V\left(t_{0}\right)+\left(\alpha^{*} \delta+\frac{1}{4} \alpha^{* 2}\right) T \lim _{i \rightarrow \infty} \sum_{j=0}^{i-1} \varepsilon^{i-j}-k_{2} \lim _{i \rightarrow \infty} \sum_{j=0}^{i-1} \int_{t-(j+1) \mathrm{T}}^{t-j T} x^{2} d \tau \\
\lim _{t \rightarrow \infty} \int_{t-T}^{t} x^{2} d \tau=0
\end{gathered}
$$

The controller design method is not only suitable for linear parameter system (12), the same also applies to nonlinear parametric system, considering the [20] of a class of norder nonlinear parametric system:

$$
\begin{aligned}
& \dot{x}_{i}=x_{i+1} \\
& \dot{x}_{n}=\frac{1}{\beta\left(X, \varphi_{\beta}\right)}\left(f\left(X, \varphi_{f}\right)+g(X, \theta(t))+u+\eta(X, t)\right) \\
& y=x_{1}
\end{aligned}
$$

In parametric system identification, a critical problem is finding a suitable structure within which a good model can be found. In non-adaptive identification, guided by varying degrees of physical information, the model form is ordinarily fixed prior to estimating parameters. The term "black-box" is used to describe a model that has been selected independently of any physical system knowledge [1]. Linear, time-invariant (LTI) models are frequently used because of their simplicity and the wealth of theoretical and algorithmic support that attends these structures. Nonlinear model identification, especially the black box type without guidance model selection is available, it is still a challenging problem. This article puts forward the methods of LTI (LTIiP) in the model parameters, but it could be very nonlinear interaction between the signals. The center of the ellipsoid can be used as a point estimate if needed and it explain the contraction to optimize RLS estimating weight selection. Several attributes can be used to infer the fitness of a specific amount of regression function set. In this article, we use the number of the super ellipsoid, and that is associated with the estimates of variance. Each chromosome is assigned a fitness value is derived from the objective function and then 
used in the new population to more suitable for individual selection bias. It is very suitable for the probability of individual selected high reproduction. The process continues through subsequent generations. Population increases the average fitness is more suitable for individual and interbreed and is not suitable for people dead. At the termination of the evolutionary selection algorithm to achieve a certain number of generations or fitness of the standard deviation of the population to reach the specified minimum, evolutionary operation, mutations, copy and replace are taken into consideration. Judging from a practical engineering point of view, the robust control is appealing because it is impossible to obtain an exact mathematical model of the concerned system without any assumption. There have been some investigations on DR focusing on the linear uncertain systems from several disparate perspectives. However, until now, no tractable global approach to the IDR of the uncertain nonlinear systems has been addressed. Indeed, because the structural property of the uncertainty is changed in discretization, it is impossible to directly apply the previous results to this problem. Moreover, to our knowledge, solving the robust stabilization problem remains an open problem in the research field of IDR

\section{Experimental Analysis}

Example 1, we adopt the inverted pendulum model:

$$
\begin{aligned}
& \dot{x}_{1}=x_{2} \\
& \dot{x}_{2}=\frac{g \sin x_{1}-\frac{m L x_{2}^{2} \sin x_{1} \cos x_{1}}{M+m}}{L\left(\frac{4}{3}-\frac{m \cos ^{2} x_{1}}{M+m}\right)}+\frac{\frac{\cos x_{1}}{M+m}}{L\left(\frac{4}{3}-\frac{m \cos ^{2} x_{1}}{M+m}\right)} u \\
& y=x_{1}
\end{aligned}
$$

Simulation results are shown in Figure 1-5. Figure 1 in implementing line shows the system actual output, dotted the ideal trajectory, after 1 seconds, the system output asymptotic tracking the ideal trajectory. The literature [33] convergence rate was obviously improved. Figure 2 shows the error convergence process, with the increase of cycles, error in lowering, after about five cycle study, stable convergence precision near 10.5, proved that the designed controller is suitable for the tracking control of nonlinear parametric system. Figure 3 and figure 4 said the change of the parameter estimation, with the passage of time, the estimate is gradually stabilized, and the cyclical, but always fluctuate within a certain scope, reflected the saturated learning law to ensure the effect of parameter estimation of bounded. Figure 5 is a periodic change of the control input.

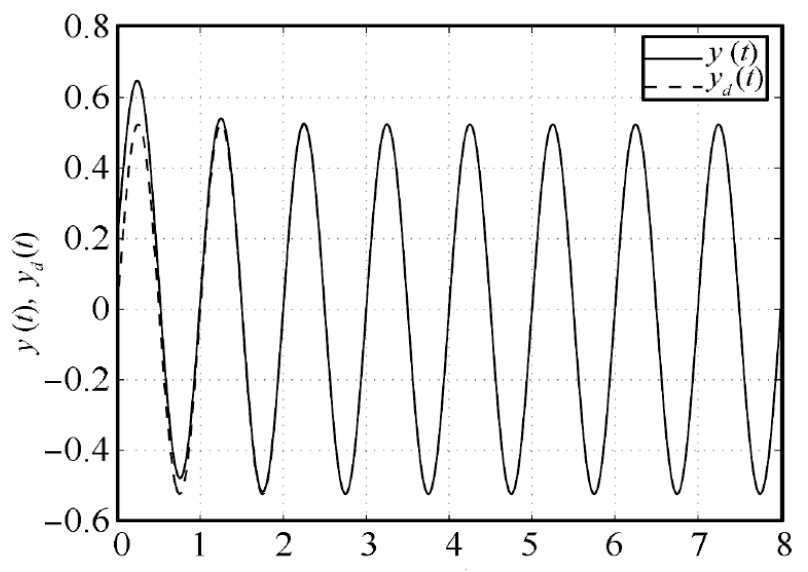

Figure 1. System Output and Ideal Trajectories 


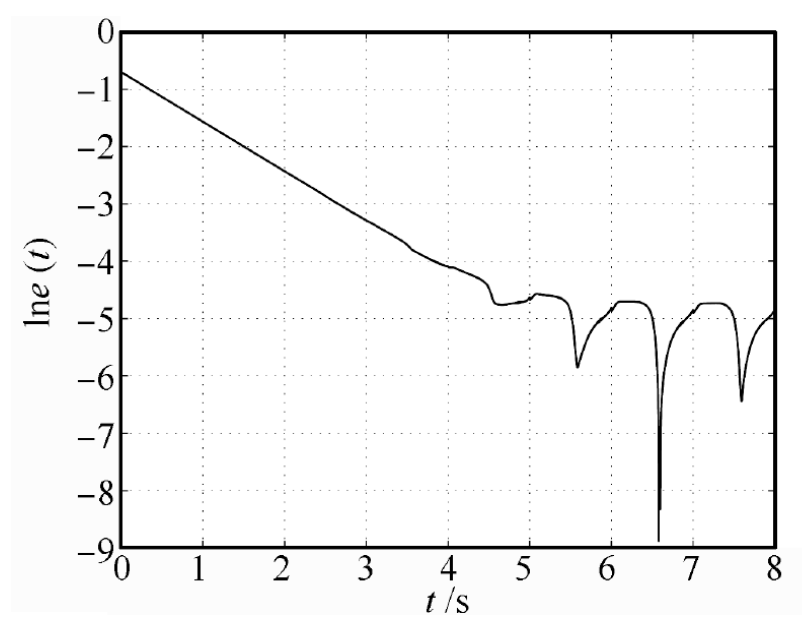

Figure 2. System Error
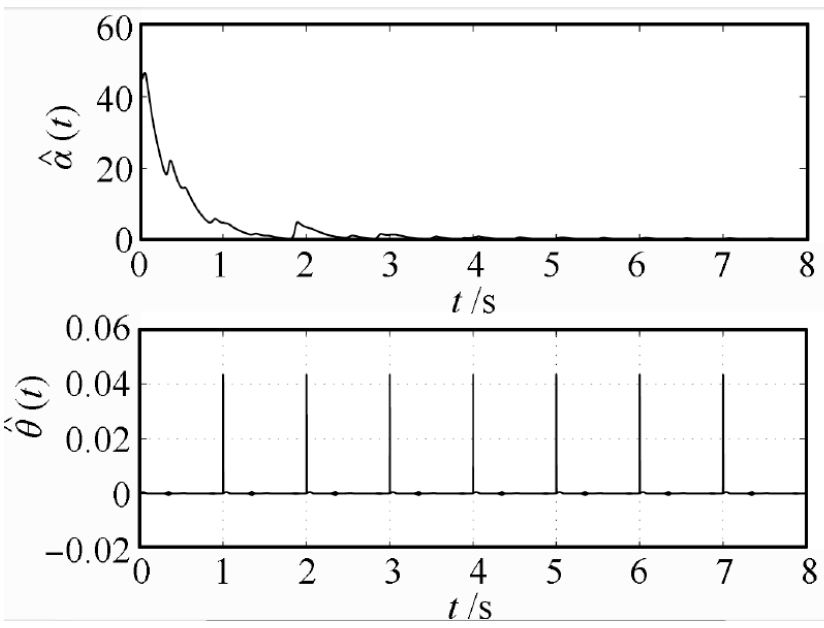

Figure 3. Parameter Estimations
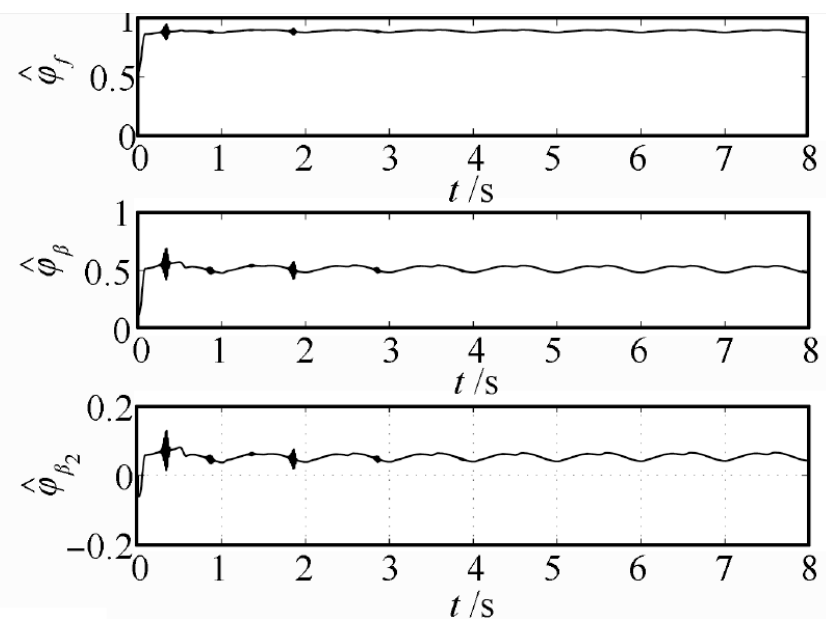

Figure 4. Parameter Estimations 


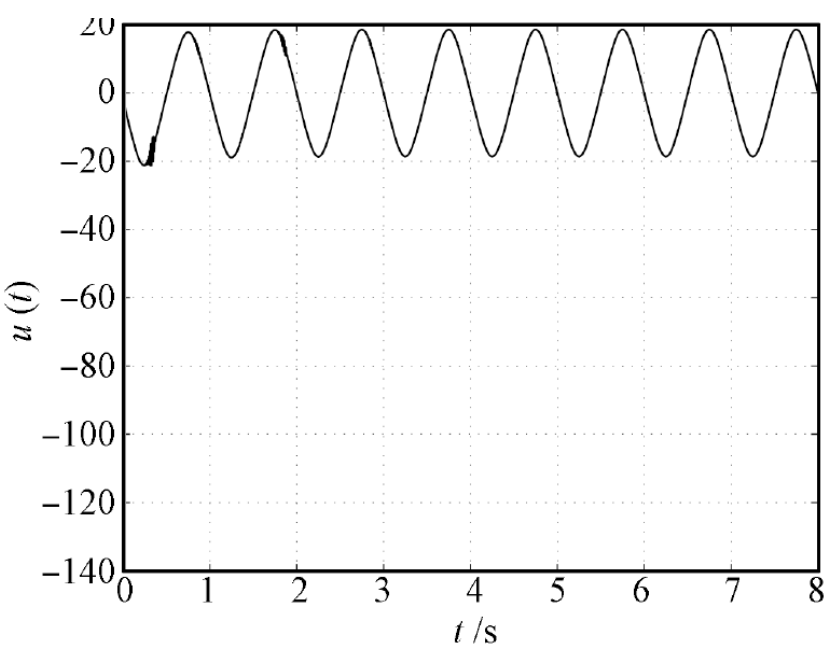

Figure 5. Control Input

The simulation results are shown in Figure 6-10. Figure 6 show the system output and the ideal trajectory, in the case of the initial error is larger, the system output after 1.5 seconds, converges to the ideal trajectory; Figure 7 shows the error precision with the change of time, after four cycles of parameter study, about 3 seconds, stable convergence precision near 10.3, literature [21] takes about 40 times of iterative learning error convergence precision can reach 10.3, so the method improved on the convergence time. Can be seen from the Figure 7, in this paper, the neural network controller design method can effectively eliminate the influence of approximation error, so as to achieve control objectives.

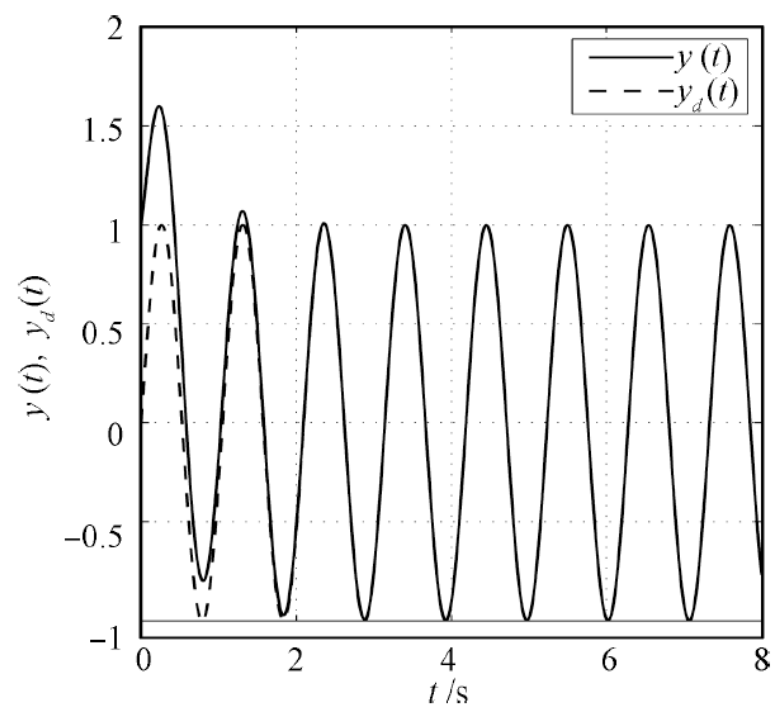

Figure 6. System Output and Ideal Trajectories 


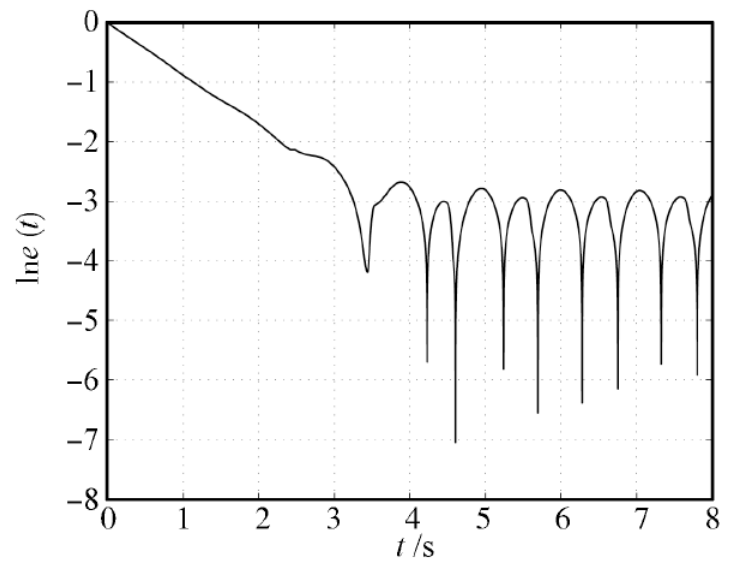

Figure 7. System Error
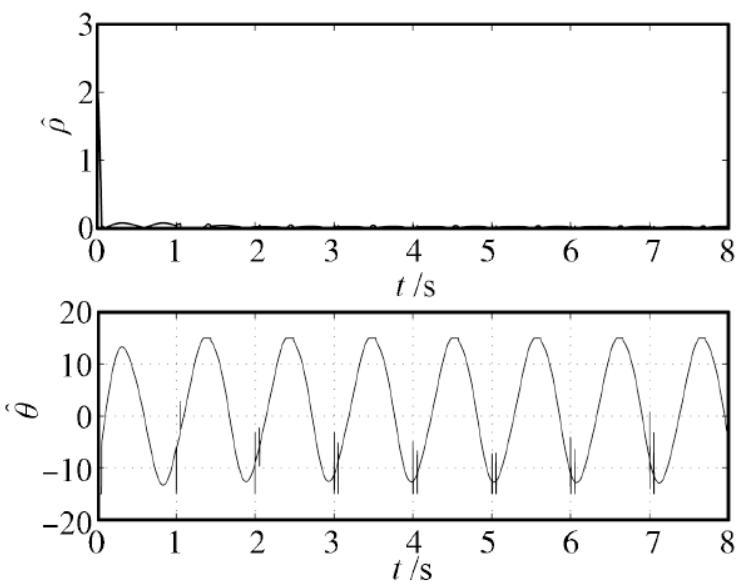

Figure 8. Parameter Estimations

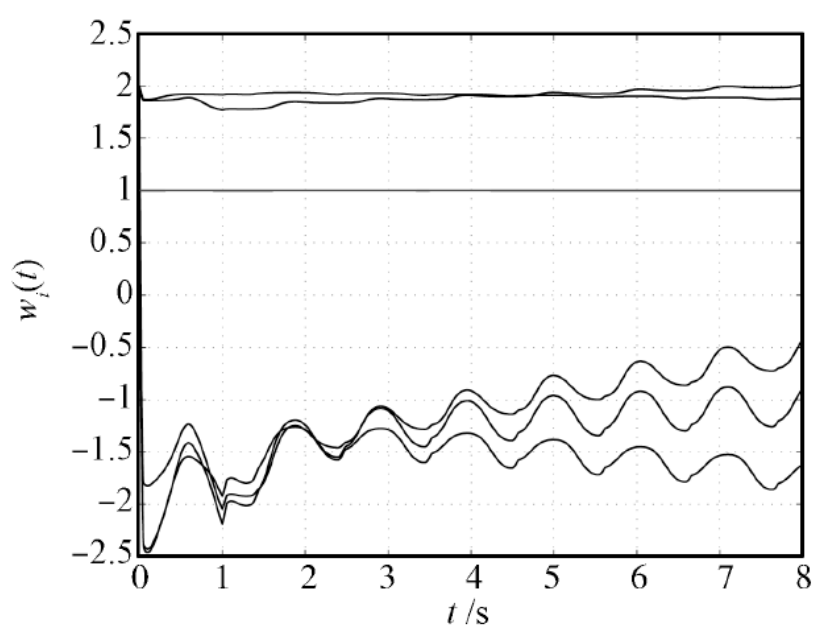

Figure 9. The Weights of Neural Network 


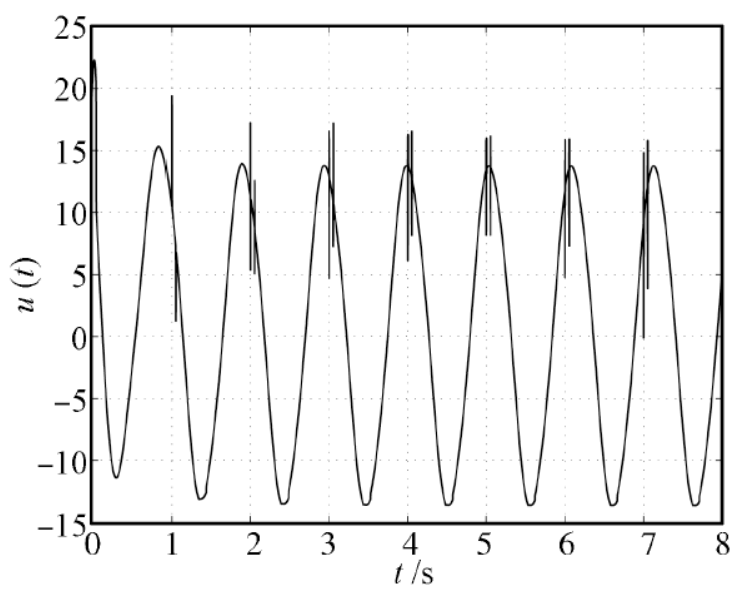

Figure 10. The Control Input

\section{Conclusions}

This paper solves the repetitive control of periodic time-varying system. In the case of the system has the aperiodic uncertainty, repeated robust adaptive controller is designed. In view of the nonlinear parametric system, on the premise of trajectory tracking cycle, the use of part of the clipping or whole limiter study law of approximate periodic timevarying function, unlike the existing repetitive control will study cycle repeated characteristics of fully integrated into the item to the robustness of the controller, make caused by uncertain disturbance error increases with the number of cycles, finally realizes the system output trajectory tracking period, and the method is used to eliminate neural network approximation error on the repetitive control system. The influence of the theory, the week periods tends to infinity, the hyperbolic function tend to be more symbolic function, the system will appear flutter phenomenon, so in actual design, by parameters adjustment to avoid the occurrence of the phenomenon. In the future, we plan to adopt optimization methodology to modify our proposed method.

\section{References}

[1] Benosman, Mouhacine. "Learning-based adaptive control for nonlinear systems." Control Conference (ECC), 2014 European. IEEE, 2014.

[2] Ding, Jian, and Huizhong Yang. "Adaptive Iterative Learning Control for a Class of Uncertain Nonlinear Systems with Second-Order Sliding Mode Technique." Circuits, Systems, and Signal Processing 33.6 (2014): 1783-1797.

[3] Hahne, J. M., et al. "Linear and Nonlinear Regression Techniques for Simultaneous and Proportional Myoelectric Control." Neural Systems and Rehabilitation Engineering, IEEE Transactions on 22.2 (2014): 269-279.

[4] Yu, Jinpeng, et al. "Neural network-based adaptive dynamic surface control for permanent magnet synchronous motors." (2014): 1-1.

[5] Liu, Derong, Ding Wang, and Hongliang Li. "Decentralized stabilization for a class of continuous-time nonlinear interconnected systems using online learning optimal control approach." (2014): 1-1.

[6] Wei, Qinglai, Derong Liu, and Yancai Xu. "Neuro-optimal tracking control for a class of discrete-time nonlinear systems via generalized value iteration adaptive dynamic programming approach." Soft Computing (2014): 1-10.

[7] Wang, Ding, et al. "Neural-network-based robust optimal control design for a class of uncertain nonlinear systems via adaptive dynamic programming." Information Sciences 282 (2014): 167-179.

[8] Smuts, Jacques Francois. A qualitative comparison between PID, adaptive and neural network control with reference to applications in drum level control on non-linear plant. Diss. 2014.

[9] $\mathrm{Li}$, Yongming, et al. "Adaptive fuzzy control of uncertain stochastic nonlinear systems with unknown dead zone using small-gain approach." Fuzzy Sets and Systems 235 (2014): 1-24.

[10] Elkoteshy, Yasser, L. C. Jiao, and Weisheng Chen. "ELM-based adaptive backstepping neural control for a class of uncertain MIMO nonlinear systems with predefined tracking accuracy." International Journal of Control 87.5 (2014): 1047-1060. 
[11] Loke, R. E., and G. Cembrano. "Neural adaptive control of a bioreactor." Intelligent Components and Instruments for Control Applications 1994 (2014): 175.

[12] Hwang, Chih-Lyang, Chiang-Cheng Chiang, and Yao-Wei Yeh. "Adaptive Fuzzy Hierarchical SlidingMode Control for the Trajectory Tracking of Uncertain Under-Actuated Nonlinear Dynamic Systems." (2014): 1-1.

[13] Subasri, R., S. Suresh, and A. M. Natarajan. "Discrete direct adaptive ELM controller for active vibration control of nonlinear base isolation buildings." Neurocomputing 129 (2014): 246-256.

[14] Zhong, Xiangnan, et al. "Optimal Control for Unknown Discrete-Time Nonlinear Markov Jump Systems Using Adaptive Dynamic Programming." (2014): 1-1.

[15] Yang, Xiong, et al. "Discrete-time online learning control for a class of unknown nonaffine nonlinear systems using reinforcement learning." Neural Networks 55 (2014): 30-41.

[16] Cui, Guozeng, et al. "Adaptive neural control of stochastic nonlinear systems with multiple time-varying delays and input saturation." Neural Computing and Applications (2014): 1-13.

[17] Yang, Xiong, Derong Liu, and Ding Wang. "Reinforcement learning for adaptive optimal control of unknown continuous-time nonlinear systems with input constraints." International Journal of Control 87.3 (2014): 553-566.

[18] Akraminia, M., M. J. Mahjoob, and M. Tatari. "Active noise control using adaptive POLYnomial Gaussian WinOwed wavelet networks." Journal of Vibration and Control (2014): 1077546313520025.

[19] Wei, Qinglai, and Derong Liu. "Neural-network-based adaptive optimal tracking control scheme for discrete-time nonlinear systems with approximation errors." Neurocomputing 149 (2015): 106-115.

[20] Shahsavari, Behrooz, et al. "Adaptive Repetitive Control Using a Modified Filtered-X LMS Algorithm." ASME 2014 Dynamic Systems and Control Conference. American Society of Mechanical Engineers, 2014.

[21] Al Janaideh, Mohammad, and Dennis S. Bernstein. "Adaptive control of Hammerstein systems with unknown Prandtl-Ishlinskii hysteresis." Proceedings of the Institution of Mechanical Engineers, Part I: Journal of Systems and Control Engineering 229.2 (2015): 149-157.

[22] Monter, Ana Rodríguez, et al. "Detailed analysis of the implementation of frequency-adaptive resonant and repetitive current controllers for grid-connected converters." Electric Power Systems Research 116 (2014): 231-242.

[23] Abidi, Khalid. "A robust discrete-time adaptive control approach for systems with almost periodic time-varying parameters." International Journal of Robust and Nonlinear Control 24.1 (2014): 166-178.

[24] $\mathrm{Na}$, Jing, et al. "Repetitive control of servo systems with time delays." Robotics and Autonomous Systems 62.3 (2014): 319-329.

[25] Ding, Jian, and Huizhong Yang. "Adaptive Iterative Learning Control for a Class of Uncertain Nonlinear Systems with Second-Order Sliding Mode Technique." Circuits, Systems, and Signal Processing 33.6 (2014): 1783-1797.

[26] Jung, J-W., et al. "A Three-Phase Inverter for a Standalone Distributed Generation System: Adaptive Voltage Control Design and Stability Analysis." (2014): 1-11.

[27] Zhang, Ruikun, et al. "Adaptive iterative learning control for a class of non-linearly parameterised systems with input saturations." International Journal of Systems Science ahead-of-print (2014): 1-11.

[28] Zhang, Ruikun, et al. "Adaptive iterative learning control for a class of non-linearly parameterised systems with input saturations." International Journal of Systems Science ahead-of-print (2014): 1-11.

[29] Sun, Yunping, et al. "Adaptive learning control for bilinear parametric time-varying systems with unknown time-varying delays." System Science and Engineering (ICSSE), 2014 IEEE International Conference on. IEEE, 2014.

[30] Wu, Zhizheng, et al. "Dynamic Head-Disk Interface Modeling and Adaptive Control of a Hybrid Actuator for Optical Data Storage Systems." International Journal of Optomechatronics ahead-of-print (2015): 1-27.

[31] Daepp, Hannes G., and Wayne J. Book. "Predictive Friction Compensation for Control of Pneumatic Actuators." 8th FPNI Ph. D Symposium on Fluid Power. American Society of Mechanical Engineers, 2014.

[32] Wang, Jing, et al. "Adaptive iterative learning control based on unfalsified strategy for Chylla-Haase reactor." Automatica Sinica, IEEE/CAA Journal of 1.4 (2014): 347-360.

[33] Zhang, Guoqing, and Xianku Zhang. "Concise adaptive neural control of uncertain nonlinear systems with periodically nonlinear time-varying parameterized disturbances." Journal of Process Control 24.4 (2014): 410-423. 